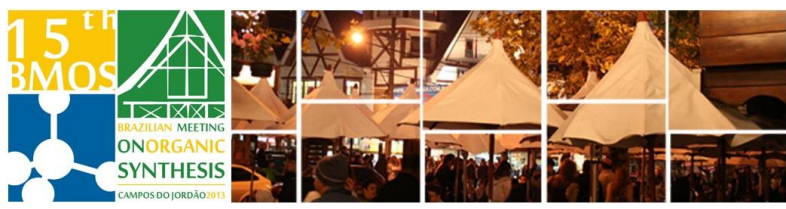

\title{
Synthesis of Dipyrone Metabolites and Evaluation of their Antipyretic Effect
}

\author{
Artur Vaz, Fernando A. Aguiar, David C. Malvar, Gloria E. P. Souza, Giuliano C. Clososki
}

Research Center for Natural and Synthetic Products, Faculty of Pharmaceutical Sciences of Ribeirão Preto University of São Paulo. artur.lara.vaz@usp.br

Keywords: synthesis, dipyrone, metabolites

\section{INTRODUCTION}

Metamizole, or dipyrone (DIP), is a widely used prodrug (bioprecursor class) with powerful analgesic and antipyretic effects. In the gastrointestinal tract DIP is first hydrolyzed to 4-methylaminoantipirine (4MAA), which is then absorbed and metabolized in the liver to the corresponding 4-aminoantipyrine (4$\mathrm{AA}$ ) and 4-formylamino-antipyrine (4-FAA) derivatives. Furthermore, the 4-AA suffers an acylation reaction to afford the 4acetylaminoantipyrine (4-AAA $)^{1}$. Despite some authors have proposed that 4-MAA and 4-AA are the active metabolites of dipyrone ${ }^{2}$, the exact mechanism of action of these and other bioactive metabolites is still unknown. Thus, in this work we have synthesized the main metabolites of dipyrone ${ }^{3}$ and these have been used in studies ${ }^{4}$ that aims to compare their antipyretic effects in the fever induced by LPS and Tsv (Tityus serrulatus venom) ${ }^{5}$.

\section{RESULTS AND DISCUSSION}

The synthetic strategies found to be most successful for the synthesis of the metabolites of interest are summarized in the figure below (Figure 1). 4Methylamino-antipyrine was obtained in $60 \%$ yield thought the basic hydrolysis of metamizole sodium 6 . On the other hand, 4-acetylaminoantipyrine and 4formylamino-antipyrine were synthesized from the commercial available 4-aminoantipyrine in 60 to $70 \%$ yields. Other strategies for the acylation and formylation of 4-AA were tested, including attempts with reacting in aqueous medium, shown in Figure 1 with chromatographic yields. Such methods, when found successful, are interesting for industrial scaleup as they bring environment-friendly advantages.

The biological activity of these synthetic individual metabolites are being accessed for the elucidation of their kinetic, therapeutic and possibly adverse effects, as well as giving insights of the roles of specific inflammation and fever mediators, i.e. Prostaglandin $\mathrm{E}_{2}$. The administration of the synthetic metabolites have shown, in animal models of LPS and Tsv-induced fever, which ones are able to reach the central nervous system and which ones are responsible for the inhibition of PGE2 synthesis the hypothalamus, thus having antipyretic and hypothermic properties.

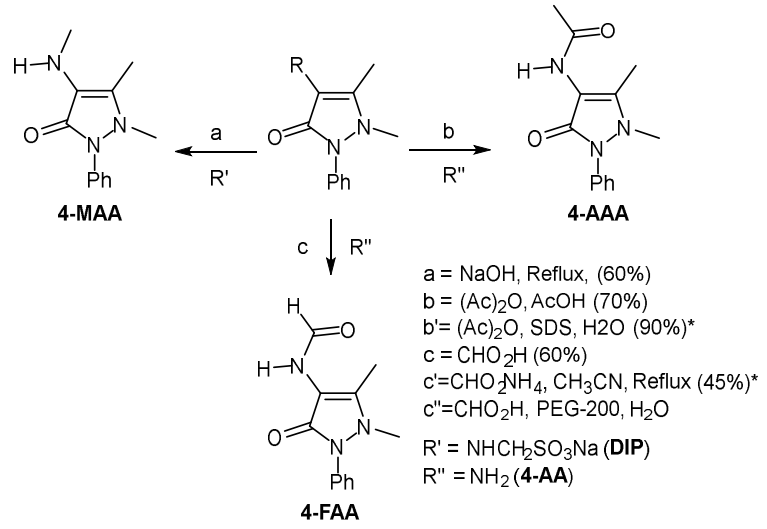

Figure 1 Schematics of Synthetic Pathways Investigated

It was shown that 4-MAA and 4-AA, but not 4-FAA or 4-AAA, are more effective in inducing antipyresis than DIP while 4-FAA only have antipyretic effect in concentrations that surpass therapeutic doses. It was also found that 4-MAA produces a dose dependent antipyretic effect on PGE2-independent fever induced by Tsv, suggesting 4-MAA, or its metabolites, is responsible for the dual antipyretic effect of dipyrone. Finally, neither 4-AA nor 4-AAA induces hypothermia suggesting that the antipyretic and hypothermic effects of dipyrone are produced by distinct metabolites.

\section{CONCLUSION}

The synthesis of DIP metabolites have been carried with success in good yield, while exploration of alternative strategies have been shown to be promising approaches in order to afford better yields with milder reacting conditions. The synthesized antipyretic compounds are being successfully utilized in pharmacokinetic and pharmacodynamic studies

\section{ACKNOWLEDGEMENTS}

\section{CAPES, CNPQ e FAPESP}

\section{REFERENCES}

1. Clin Pharmacol Ther. 58:198, 1995.

2. FASEB J. 21:2343, 2007

3. Eur. J. Pharm. Sci. 28: 15, 2006.

4. G.E.P. Souza and col., unpublished results.

5. Chem. Med. Chem. 3:1956, 2008.

6. European Journal of Medicinal Chemistry 46:4566 4572, 2011 